\title{
The Cosmology of Modified Gauss-Bonnet Gravity
}

\author{
Baojiu Li, ,, J John D. Barrow,, , \\ ${ }^{1}$ Department of Applied Mathematics and Theoretical Physics, \\ Centre for Mathematical Sciences, Wilberforce Road, \\ University of Cambridge, Cambridge CB3 OWA, United Kingdom \\ ${ }^{2}$ Institute of Theoretical Physics, University of Heidelberg, 69120 Heidelberg, Germany
}

(Dated: February 11, 2013)

\begin{abstract}
We consider the cosmology where some function $f(G)$ of the Gauss-Bonnet term $G$ is added to the gravitational action to account for the late-time accelerating expansion of the universe. The covariant and gauge invariant perturbation equations are derived with a method which could also be applied to general $f\left(R, R^{a b} R_{a b}, R^{a b c d} R_{a b c d}\right)$ gravitational theories. It is pointed out that, despite their fourthorder character, such $f(G)$ gravity models generally cannot reproduce arbitrary background cosmic evolutions; for example, the standard $\Lambda$ CDM paradigm with $\Omega_{\mathrm{DE}}=0.76$ cannot be realized in $f(G)$ gravity theories unless $f$ is a true cosmological constant because it imposes exclusionary constraints on the form of $f(G)$. We analyze the perturbation equations and find that, as in $f(R)$ model, the stability of early-time perturbation growth puts some constraints on the functional form of $f(G)$, in this case $\partial^{2} f / \partial G^{2}<0$. Furthermore, the stability of small-scale perturbations also requires that $f$ not deviate significantly from a constant. These analyses are illustrated by numerically propagating the perturbation equations with a specific model reproducing a representative $\Lambda$ CDM cosmic history. Our results show how the $f(G)$ models are highly constrained by cosmological data.
\end{abstract}

PACS numbers: 04.50.+h

Keywords:

\section{INTRODUCTION}

The accumulating astronomical evidence for the present accelerating expansion of the universe has stimulated many investigations into the nature of the dark energy which might be responsible for this unexpected dynamics (for a review see, e.g., [1]). Besides proposing to add some kinds of exotic (and purely theoretical) matter species into the energy budget of the universe, many investigators have also focused their attentions on modifying general relativity (GR) on the largest scales so as to introduce significant modifications at late times. One example is provided by the family of $f(R)$ gravity models, which had also been considered before the discovery of cosmic acceleration (see for example Refs. 2, 3, 4] ). In Refs. [5, 6], the authors discuss a specific model where the correction to GR is a polynomial function of the $R^{2}, R^{a b} R_{a b}$ and $R^{a b c d} R_{a b c d}$ quadratic curvature invariants (here, $R, R_{a b}$ and $R_{a b c d}$ are respectively the Ricci scalar, Ricci tensor and Riemann tensor) and showed that there exist late-time accelerating attractors in Friedmann cosmological solutions to the theory. It is very interesting that when the Ricci scalar $R$ in the Einstein-Hilbert action is replaced by some general functions of $R$ and $R^{a b} R_{a b}$, it becomes necessary to distinguish between two different variational approaches in deriving the field equations. In the metric approach, as in Refs. [5, [6], the metric components $g_{a b}$ are the only variational variables and

\footnotetext{
*Email address: B.Li@damtp.cam.ac.uk

${ }^{\dagger}$ Email address: J.D.Barrow@damtp.cam.ac.uk

${ }^{\ddagger}$ Email address: d.mota@thphys.uni-heidelberg.de
}

the field equations are generally of fourth-order, which makes the theories phenomenologically richer but more stringently constrained in many cases. Within the Palatini variational approach, on the other hand, we treat the metric $g_{a b}$ and connection $\Gamma_{b c}^{a}$ as independent variables and extremize the action with respect to both of them, and the resulting field equations are second order and easier to solve. The Palatini $f(R)$ gravity is also proposed as an alternative to dark energy in a series of works [7, 8, 9, 10]. There has since been growing interest in these modified gravity theories: for the local tests of the Palatini and metric $f(R)$ gravity models see [11, 12], and for the cosmologies of these two classes of models see [13, 14, 15, 16, 17, 18, 19].

Both approaches to modifying gravity are far from problem-free. In the metric $f(R)$ gravity models, the theory is conformally related to standard GR plus a selfinteracting scalar field [3], which generally introduces extra forces inconsistent with solar system tests [12]. The Palatini approach, on the other hand, generally leads to a large (or even negative) sound-speed-squared term in the growth equation of the matter perturbations on small scales, and its predicted shapes of the cosmic microwave background $(\mathrm{CMB})$ and matter power-spectra deviate unacceptably from those predicted in GR [17, 18, 19], and so fail the most fundamental cosmological tests. Again, these examples reiterate the difficulties encountered when trying to make general modified gravity theories compatible with observations.

In this work we will focus on another form of modified gravity, the modified Gauss-Bonnet theory, which is proposed and discussed in [20, 21, 22, 23, 24] (see, for example, 25, 26, 27] for a related model). In these 
models, GR is modified by adding an arbitrary function $f(G)$ into the gravitational action, where $G \equiv$ $R^{2}-4 R^{a b} R_{a b}+R^{a b c d} R_{a b c d}$ is the Gauss-Bonnet invariant (which is a topological invariant in four dimensions). Such correction is motivated by the effective low-energy actions in string theory and is shown to be able to pass solar-system tests even though it arises in the metric variational approach. Furthermore, refs. [20, 23] also demonstrate that such models can produce late-time cosmic acceleration, as well as a transition from deceleration to acceleration, or from a non-phantom phase to a phantom phase. Here, we are interested in the perturbation dynamics in such modified gravity theories, and the cosmology arising from them at first-order in perturbation theory.

Our presentation is organized as follows. In Sec. II we briefly introduce the model and present the perturbation equations of general $f(G)$ model in covariant and gauge invariant (CGI) form. For this, we generalize the effective energy-momentum tensor approach of deriving CGI modified gravitational field equations in a way which could be applied to a general $f\left(R, R^{a b} R_{a b}, R^{a b c d} R_{a b c d}\right)$ theory - which includes the $f(G)$ and $f(R)$ models as specific cases. In Sec. III we shall discuss the background evolution of $f(G)$ models and explain why arbitrary cosmic histories cannot be realized with the $f(G)$ model. The perturbation equations are then analyzed in Sec. IV] where we also evolve these equations numerically to investigate the $f(G)$ effects on the growth of linear perturbations. Our discussion and conclusions are presented in Sec. D. Throughout this work our convention is chosen as $\left[\nabla_{a}, \nabla_{b}\right] u^{c}=R_{a b d}^{c} u^{d}, R_{a b}=R_{a c b}{ }^{c}$ where $a, b, \cdots$ run over $0,1,2,3$ and $c=\hbar=1$; the metric signature is $(+,-,-,-)$ and the universe is assumed to be spatially flat and filled with photons, baryons, cold dark matter (CDM) and three species of effectively massless neutrinos.

\section{FIELD EQUATIONS IN MODIFIED GAUSS-BONNET GRAVITY}

In this section we briefly introduce the main ingredients of $f(G)$ gravity and derive the general perturbation equations that govern the dynamics of small inhomogeneities in the cosmological models that arise in this theory.

\section{A. The Generalized Einstein Equations}

Our starting point for $f(G)$ gravity is the modified Einstein-Hilbert action,

$$
S=\int d^{4} x \sqrt{-g}\left[\frac{R+f(G)}{2 \kappa}+\mathcal{L}_{m}\right],
$$

in which $\kappa=8 \pi G_{\mathrm{N}}$, with $G_{\mathrm{N}}$ being the gravitational constant and $R=R\left(g_{a b}\right)$ is the Ricci scalar. Varying this action with respect to the metric $g_{a b}$ gives the modified Einstein equations

$$
\begin{aligned}
& R_{a b}-\frac{1}{2} g_{a b} R \\
= & \kappa T_{a b}^{f}+\frac{1}{2} g_{a b} f-2 F R R_{a b}+4 F R_{a}^{c} R_{b c} \\
& -2 F R_{a c d e} R_{b}^{c d e}-4 F R_{a c d b} R^{c d}+2 R \nabla_{a} \nabla_{b} F \\
& -2 R g_{a b} \nabla^{2} F-4 R_{a}^{c} \nabla_{b} \nabla_{c} F-4 R_{b}^{c} \nabla_{a} \nabla_{c} F \\
& +4 R_{a b} \nabla^{2} F+4 g_{a b} R^{c d} \nabla_{c} \nabla_{d} F-4 R_{a c b d} \nabla^{c} \nabla^{d} F,(2)
\end{aligned}
$$

where $F=F(G) \equiv \partial f(G) / \partial G$ (notice that, unlike in the $f(R)$ models, $F$ here is not dimensionless) and $T_{a b}^{f}$ is the energy-momentum tensor of the fluid matter (photons, baryons, cold dark matter, and light neutrinos). The trace of Eq. (2) reads

$$
\begin{aligned}
-R= & \kappa\left(\rho^{f}-3 p^{f}\right)+2 f-2 F G \\
& -2 R \square F+4 R_{a b} \nabla^{a} \nabla^{b} F,
\end{aligned}
$$

with $T_{a}^{a} \equiv T=\rho^{f}-3 p^{f}$. We see that the curvaturerelated quantities $(R, G, F, \cdots)$ are determined by the energy-momentum tensor of the fluid matter through a complicated dynamical equation Eq. (3) and thus the modification to the GR field equations can be understood as a change in the way that the spacetime curvature, and thus the Ricci tensor $R_{a b}$, responds to the distribution of matter.

\section{B. The Perturbation Equations in General Relativity}

The CGI perturbation equations in general theories of $f(G)$ gravity are derived in this section using the method of $3+1$ decomposition [28, 29, 30, 31]. Furthermore, we shall adopt the effective energy-momentum tensor approach [16, 32], which treats the modifications on the right hand side of Eq. (2) as an effective energymomentum tensor. However, since the modification generally involves terms nonlinear in $R_{a b}, R_{a b c d}$, we should express these terms appropriately. This will be done in more detail below, but now let us briefly review the main ingredients of $3+1$ decomposition and their application to standard general relativity for ease of later reference (as in GR, there is only fluid matter so we shall neglect the superscript $f$ in this subsection).

The main idea of $3+1$ decomposition is to make spacetime splits of physical quantities with respect to the 4velocity $u^{a}$ of an observer. The projection tensor $h_{a b}$ is defined as $h_{a b}=g_{a b}-u_{a} u_{b}$ and can be used to obtain covariant tensors perpendicular to $u$. For example, the covariant spatial derivative $\hat{\nabla}$ of a tensor field $T_{d \cdots e}^{b \cdots c}$ is defined as

$$
\hat{\nabla}^{a} T_{d \cdots e}^{b \cdots c} \equiv h_{i}^{a} h_{j}^{b} \cdots h_{k}^{c} h_{d}^{r} \cdots h_{e}^{s} \nabla^{i} T_{r \cdots s}^{j \cdots k} .
$$

The energy-momentum tensor and covariant derivative 
of the 4-velocity are decomposed respectively as

$$
\begin{aligned}
T_{a b} & =\pi_{a b}+2 q_{(a} u_{b)}+\rho u_{a} u_{b}-p h_{a b}, \\
\nabla_{a} u_{b} & =\sigma_{a b}+\varpi_{a b}+\frac{1}{3} \theta h_{a b}+u_{a} A_{b} .
\end{aligned}
$$

In the above, $\pi_{a b}$ is the projected symmetric trace-free (PSTF) anisotropic stress, $q_{a}$ the vector heat flux vector, $p$ the isotropic pressure, $\sigma_{a b}$ the PSTF shear tensor, $\varpi_{a b}=\hat{\nabla}_{[a} u_{b]}$, the vorticity, $\theta=\nabla^{c} u_{c}=3 \dot{a} / a$ ( $a$ is the mean expansion scale factor) the expansion scalar, and $A_{b}=\dot{u}_{b}$ the acceleration; the overdot denotes time derivative expressed as $\dot{\phi}=u^{a} \nabla_{a} \phi$, brackets mean antisymmetrisation, and parentheses symmetrization. The normalization is chosen to be $u^{a} u_{a}=1$. The quantities $\pi_{a b}, q_{a}, \rho, p$ are referred to as dynamical quantities and $\sigma_{a b}, \varpi_{a b}, \theta, A_{a}$ as kinematical quantities. Note that the dynamical quantities can be obtained from the energymomentum tensor $T_{a b}$ through the relations

$$
\begin{aligned}
\rho & =T_{a b} u^{a} u^{b}, \\
p & =-\frac{1}{3} h^{a b} T_{a b}, \\
q_{a} & =h_{a}^{d} u^{c} T_{c d}, \\
\pi_{a b} & =h_{a}^{c} h_{b}^{d} T_{c d}+p h_{a b} .
\end{aligned}
$$

Decomposing the Riemann tensor and making use the Einstein equations, we obtain, after linearization, five constraint equations [30, 31]:

$$
\begin{aligned}
0 & =\hat{\nabla}^{c}\left(\varepsilon_{c d}^{a b} u^{d} \varpi_{a b}\right) ; \\
\kappa q_{a} & =-\frac{2 \hat{\nabla}_{a} \theta}{3}+\hat{\nabla}^{b} \sigma_{a b}+\hat{\nabla}^{b} \varpi_{a b} ; \\
\mathcal{B}_{a b} & =\left[\hat{\nabla}^{c} \sigma_{d(a}+\hat{\nabla}^{c} \varpi_{d(a}\right] \varepsilon_{b) e c}{ }^{d} u^{e} ; \\
\hat{\nabla}^{b} \mathcal{E}_{a b} & =\frac{1}{2} \kappa\left[\hat{\nabla}^{b} \pi_{a b}+\frac{2}{3} \theta q_{a}+\frac{2}{3} \hat{\nabla}_{a} \rho\right] ; \\
\hat{\nabla}^{b} \mathcal{B}_{a b} & =\frac{1}{2} \kappa\left[\hat{\nabla}_{c} q_{d}+(\rho+p) \varpi_{c d}\right] \varepsilon_{a b}^{c d} u^{b},
\end{aligned}
$$

and five propagation equations,

$$
\begin{aligned}
& \dot{\theta}+\frac{1}{3} \theta^{2}-\hat{\nabla}^{a} A_{a}+\frac{\kappa}{2}(\rho+3 p)=0 ;(13) \\
& \dot{\sigma}_{a b}+\frac{2}{3} \theta \sigma_{a b}-\hat{\nabla}_{\langle a} A_{b\rangle}+\mathcal{E}_{a b}+\frac{1}{2} \kappa \pi_{a b}=0 ;(14) \\
& \dot{\varpi}+\frac{2}{3} \theta \varpi-\hat{\nabla}_{[a} A_{b]}=0 ;(15) \\
& \frac{1}{2} \kappa\left[\dot{\pi}_{a b}+\frac{1}{3} \theta \pi_{a b}\right]-\frac{1}{2} \kappa\left[(\rho+p) \sigma_{a b}+\hat{\nabla}_{\langle a} q_{b\rangle}\right]
\end{aligned}
$$

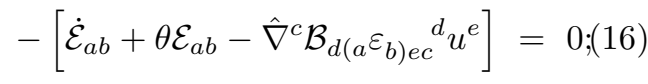

$$
\begin{aligned}
& \dot{\mathcal{B}}_{a b}+\theta \mathcal{B}_{a b}+\hat{\nabla}^{c} \mathcal{E}_{d\left({ }_{a} \varepsilon_{b) e c}\right.}{ }^{d} u^{e} \\
& +\frac{\kappa}{2} \hat{\nabla}^{c} \pi_{d(a} \varepsilon_{b) e c}{ }^{d} u^{e}=0 .(17)
\end{aligned}
$$

Here, $\varepsilon_{a b c d}$ is the covariant permutation tensor, $\mathcal{E}_{a b}$ and $\mathcal{B}_{a b}$ are respectively the electric and magnetic parts of the Weyl tensor $\mathcal{W}_{a b c d}$, defined by $\mathcal{E}_{a b}=u^{c} u^{d} \mathcal{W}_{a c b d}$ and $\mathcal{B}_{a b}=-\frac{1}{2} u^{c} u^{d} \varepsilon_{a c}{ }^{e f} \mathcal{W}_{e f b d}$. The angle bracket means taking the trace-free part of a quantity.

Besides the above equations, it is useful to express the projected Ricci scalar $\hat{R}$ into the hypersurfaces orthogonal to $u^{a}$ as

$$
\hat{R} \doteq 2 \kappa \rho-\frac{2}{3} \theta^{2}
$$

The spatial derivative of the projected Ricci scalar, $\eta_{a} \equiv$ $\frac{1}{2} a \hat{\nabla}{ }_{a} \hat{R}$, is then given as

$$
\eta_{a}=\kappa \hat{\nabla}_{a} \rho-\frac{2 a}{3} \theta \hat{\nabla}_{a} \theta
$$

and its propagation equation by

$$
\dot{\eta}_{a}+\frac{2 \theta}{3} \eta_{a}=-\frac{2}{3} \theta a \hat{\nabla}_{a} \hat{\nabla} \cdot A-a \kappa \hat{\nabla}_{a} \hat{\nabla} \cdot q .
$$

Finally, there are the conservation equations for the energy-momentum tensor:

$$
\begin{aligned}
\dot{\rho}+(\rho+p) \theta+\hat{\nabla}^{a} q_{a} & =0 \\
\dot{q}_{a}+\frac{4}{3} \theta q_{a}+(\rho+p) A_{a}-\hat{\nabla}_{a} p+\hat{\nabla}^{b} \pi_{a b} & =0 .
\end{aligned}
$$

As we are considering a spatially-flat universe, the spatial curvature must vanish on large scales and so $\hat{R}=0$. Thus, from Eq. (18), we obtain

$$
\frac{1}{3} \theta^{2}=\kappa \rho
$$

This is the Friedmann equation in standard general relativity, and the other background equations (the Raychaudhuri equation and the energy-conservation equation) are obtained by taking the zero-order parts of Eqs. (13, 21), as

$$
\begin{aligned}
\dot{\theta}+\frac{1}{3} \theta^{2}+\frac{\kappa}{2}(\rho+3 p) & =0 \\
\dot{\rho}+(\rho+p) \theta & =0 .
\end{aligned}
$$

In what follows, we will only consider scalar modes of perturbations, for which the vorticity $\varpi_{a b}$ and magnetic part of Weyl tensor $\mathcal{B}_{a b}$ are at most of second order 30, 31] and will be neglected from our first-order analysis.

\section{The Perturbation Equations in $f(G)$ Gravity}

In the effective energy-momentum tensor approach, the field equations Eqs. (8 - 25) listed above preserve their forms, but the dynamical quantities $\rho, p, q_{a}, \pi_{a b}$ appearing there should be replaced with the effective total ones $\rho^{\text {tot }}=\rho^{f}+\rho^{\mathrm{G}}, p^{\text {tot }}=p^{f}+p^{\mathrm{G}}, q_{a}^{\text {tot }}=q_{a}^{f}+q_{a}^{\mathrm{G}}, \pi_{a b}^{\text {tot }}=$ $\pi_{a b}^{f}+\pi_{a b}^{\mathrm{G}}$, in which a superscript ${ }^{\mathrm{G}}$ means the contribution from the Gauss-Bonnet correction. 
Writing the modified Einstein equations, Eq. (2) in the following form,

$$
\begin{aligned}
R_{a b}-\frac{1}{2} g_{a b} R & =\kappa T_{a b}^{\mathrm{tot}} \\
& =\kappa T_{a b}^{f}+\kappa T_{a b}^{\mathrm{G}},
\end{aligned}
$$

one can easily identify

$$
\begin{aligned}
\kappa T_{a b}^{\mathrm{G}}= & \frac{1}{2} g_{a b} f-2 F R R_{a b}+4 F R_{a}^{c} R_{b c} \\
& -2 F R_{a c d e} R_{b}^{c d e}-4 F R_{a c d b} R^{c d}+2 R \nabla_{a} \nabla_{b} F \\
& -2 R g_{a b} \nabla^{2} F-4 R_{a}^{c} \nabla_{b} \nabla_{c} F-4 R_{b}^{c} \nabla_{a} \nabla_{c} F \\
& +4 R_{a b} \nabla^{2} F+4 g_{a b} R^{c d} \nabla_{c} \nabla_{d} F-4 R_{a c b d} \nabla^{c} \nabla^{d} F
\end{aligned}
$$

and Eq. (7) can be used to calculate $\rho^{\mathrm{G}}, p^{\mathrm{G}}, q_{a}^{\mathrm{G}}, \pi_{a b}^{\mathrm{G}}$. In order to do this we need the explicit expressions for $R_{a b}$ and $R_{a b c d}$, which could be obtained in terms of either (effective total) dynamical quantities or kinematical quantities, or a mixture of the two.

To express $R_{a b}$ and $R_{a b c d}$ explicitly, now decompose the symmetric Ricci tensor $R_{a b}$ in the following general way,

$$
R_{a b}=\Delta u_{a} u_{b}+\Xi h_{a b}+2 u_{(a} \Upsilon_{b)}+\Sigma_{a b},
$$

then Eq. (26) gives

$$
\begin{aligned}
\Delta & =\frac{1}{2} \kappa\left(\rho^{\mathrm{tot}}+3 p^{\mathrm{tot}}\right) \\
& =-\left[\dot{\theta}+\frac{1}{3} \theta^{2}-\hat{\nabla}^{a} A_{a}\right] ; \\
\Xi & =-\frac{1}{2} \kappa\left(\rho^{\mathrm{tot}}-p^{\mathrm{tot}}\right) \\
& =-\frac{1}{3}\left[\dot{\theta}+\theta^{2}+\hat{R}-\hat{\nabla}^{a} A_{a}\right] ; \\
\Upsilon_{a} & =\kappa q_{a}^{\mathrm{tot}} \\
& =-\frac{2 \hat{\nabla}_{a} \theta}{3}+\hat{\nabla}^{b} \sigma_{a b}+\hat{\nabla}^{b} \varpi_{a b} ; \\
\Sigma_{a b} & =\kappa \pi_{a b}^{\mathrm{tot}} \\
& =-2\left[\dot{\sigma}_{a b}+\frac{2}{3} \theta \sigma_{a b}-\hat{\nabla}_{\langle a} A_{b\rangle}+\mathcal{E}_{a b}\right]
\end{aligned}
$$

in which we have used Eqs. (9, 13, 14, 18). Notice that the first lines are expressed in terms of total dynamical quantities and the second lines of kinematical quantities. For those terms involving $R_{a b c d}$, we shall use the decomposition of Riemann tensor extensively (keeping in mind that $\left.u^{c} u^{d} \mathcal{W}_{a c b d}=\mathcal{E}_{a b}\right)$ :

$$
\begin{aligned}
R_{a b c d}= & \frac{1}{2}\left(g_{a c} R_{b d}+g_{b d} R_{a c}-g_{a d} R_{b c}-g_{b c} R_{a d}\right) \\
& +\mathcal{W}_{a b c d}-\frac{1}{6} R\left(g_{a c} g_{b d}-g_{a d} g_{b c}\right) .
\end{aligned}
$$

For example, it is easy to show that, up to first order,

$$
\begin{aligned}
& R_{a}^{c d e} R_{b c d e} \\
= & -\frac{4}{3} \dot{\theta} \mathcal{E}_{a b}+\frac{1}{2} g_{a b} R^{c d} R_{c d}+\frac{1}{3} R R_{a b}-\frac{1}{6} g_{a b} R^{2},
\end{aligned}
$$

so that

$$
\begin{aligned}
R^{a b c d} R_{a b c d} & =2 R^{a b} R_{a b}-\frac{1}{3} R^{2} \\
G & =\frac{2}{3} R^{2}-2 R^{a b} R_{a b}
\end{aligned}
$$

where

$$
\begin{aligned}
R= & -2 \dot{\theta}-\frac{4}{3} \theta^{2}+2 \hat{\nabla}^{a} A_{a}-\hat{R} \\
R^{a b} R_{a b}= & \frac{4}{3}\left[\dot{\theta}^{2}+\dot{\theta} \theta^{2}+\frac{1}{3} \theta^{4}\right] \\
& +\frac{2}{3}\left(\dot{\theta}+\theta^{2}\right) \hat{R}-\frac{8}{3}\left[\dot{\theta}+\frac{1}{2} \theta^{2}\right] \hat{\nabla}^{a} A_{a} .
\end{aligned}
$$

With these useful relations and some calculation, the contribution to the energy-momentum tensor from the Gauss-Bonnet correction term can be identified as

$$
\begin{aligned}
\kappa \rho^{\mathrm{G}}= & \frac{1}{2}(f-F G)+\frac{2}{3}(\Delta-3 \Xi) \dot{F} \theta \\
& +\frac{2}{3}(\Delta-3 \Xi) \hat{\nabla}^{2} F \\
-\kappa p^{\mathrm{G}}= & \frac{1}{2}(f-F G)+\frac{2}{3}(\Delta-3 \Xi) \ddot{F} \\
& -\frac{8}{9} \Delta\left(\theta \dot{F}+\hat{\nabla}^{2} F\right) ; \\
\kappa q_{a}^{\mathrm{G}}= & -\frac{2}{3}(\Delta-3 \Xi)\left(\hat{\nabla}_{a} \dot{F}-\frac{1}{3} \theta \hat{\nabla}_{a} F\right)+\frac{4}{3} \dot{F} \theta \Upsilon_{a}(335) \\
\kappa \pi_{a b}^{\mathrm{G}}= & \frac{4}{3} \Delta\left(\dot{F} \sigma_{a b}+\hat{\nabla}_{\langle a} \hat{\nabla}_{b\rangle} F\right)+2\left(\ddot{F}+\frac{1}{3} \theta \dot{F}\right) \Sigma_{a b} \\
& -4 \ddot{F} \mathcal{E}_{a b}+\frac{4}{3} \theta \dot{F} \mathcal{E}_{a b} .
\end{aligned}
$$

Here, we want to make some comments about these equations. First, if $f$ is constant, then $F$ and its derivatives vanish, so that $\kappa \rho^{\mathrm{G}}=-\kappa p^{\mathrm{G}}=\frac{f}{2}$ and $\kappa q_{a}^{\mathrm{G}}=\kappa \pi_{a b}^{\mathrm{G}}=0$, and thus we have the $\Lambda \mathrm{CDM}$ limit. Second, it is not difficult to check that the above quantities satisfy the independent energy-momentum conservation equations

$$
\begin{aligned}
\dot{\rho}^{\mathrm{G}}+\left(\rho^{\mathrm{G}}+p^{\mathrm{G}}\right) \theta+\hat{\nabla}^{a} q_{a}^{\mathrm{G}}=0, \\
\dot{q}_{a}^{\mathrm{G}}+\frac{4}{3} \theta q_{a}^{\mathrm{G}}+\left(\rho^{\mathrm{G}}+p^{\mathrm{G}}\right) A_{a}-\hat{\nabla}_{a} p^{\mathrm{G}}+\hat{\nabla}^{b} \pi_{a b}^{\mathrm{G}}=0 .
\end{aligned}
$$

This is a result of the energy-momentum conservation in fluid matter and the contracted Bianchi identity. Thirdly, it would be convenient to use $\Upsilon_{a}=\kappa\left(q_{a}^{f}+q_{a}^{\mathrm{G}}\right)$ and $\Sigma_{a b}=\kappa\left(\pi_{a b}^{f}+\pi_{a b}^{\mathrm{G}}\right)$ (see Eq. (28) $)$ to rewrite Eqs. (35, 36) so that $\kappa q_{a}^{\mathrm{G}}, \kappa \pi_{a b}^{\mathrm{G}}$ are expressed respectively in terms of $\kappa q_{a}^{f}, \kappa \pi_{a b}^{f}$ and other quantities. This is what we do in the numerical calculation. Fourthly, it is interesting to note that there is no $\hat{\nabla}_{a} \dot{\theta}$ term in $\kappa \hat{\nabla}_{a} \rho^{\mathrm{G}}$ up to first order because $\Delta-3 \Xi=\frac{2}{3} \theta^{2}+\hat{R}$ and $\hat{\nabla}_{a}(f-F G)=-G \hat{\nabla}_{a} F$; this is positive because otherwise Eq. (19) will no longer be an algebraic equation for $\hat{\nabla}_{a} \theta$. For similar reasons Eq. (20) remains a first-order differential equation for $\eta_{a}$ for the present model. These simplifications also 
occur in the $f(R)$ gravity models but not in general $f\left(R, R^{a b} R_{a b}, R^{a b c d} R_{a b c d}\right)$ theories. In the later case, the method we use here to derive the CGI perturbation equations still applies and the perturbation equations will become even higher order and more complicated (specifically, some of the perturbation equations above will become propagation equations for $q_{a}^{\mathrm{MG}}$ and $\pi_{a b}^{\mathrm{MG}}$, where ${ }^{\mathrm{MG}}$ denotes general modified gravity theory [33, 34] ). Finally, we can see that the quantity $F$ here appears only to (at least directly) influence background evolutions, and with the background fixed it is its derivative which determines the perturbation evolutions.

\section{THE BACKGROUND EVOLUTION IN $f(G)$ MODELS}

In this section we discuss the background evolution in general $f(G)$ gravity models. Recall that in $f(R)$ gravity theories the fourth-order nature of the Friedmann equation allows enough freedom for the model to reproduce an arbitrary background cosmic evolution. Since the field equations are also fourth order in the $f(G)$ models, one might think that they could also describe arbitrarily parameterized background histories. However, this is not the case, as we shall see below. This is because $\dot{G}$ must change its sign in the recent past in many fixedbackground models.

For the background evolution, we use the Friedmann equation Eq. (23) with the dark energy density given by Eq. (33)

$$
\kappa \rho_{\mathrm{DE}}=\frac{1}{2} f-\frac{4}{9} \theta^{2}\left(\dot{\theta}+\frac{1}{3} \theta^{2}\right) F+\frac{4}{9} \theta^{3} \dot{F}
$$

where we have kept terms only up to zero order and used the expression $G=\frac{8}{9} \theta^{2} \dot{\theta}+\frac{8}{27} \theta^{4}$, which is obtained from Eqs. (27, 28, 31). Following [15], we define the following dimensionless quantities (here $H=\theta / 3$ is the Hubble rate and $H_{0}$ is its present-day value)

$$
\begin{aligned}
E & \equiv \frac{H^{2}}{H_{0}^{2}}, \\
y & \equiv \frac{f}{H_{0}^{2}},
\end{aligned}
$$

in terms of which $G$, and so Eq. (37) can be written as

$$
G=12 H_{0}^{4} E\left(E^{*}+2 E\right)
$$

with

$$
\begin{aligned}
& y^{* *}-\left(\frac{G}{24 E^{2}}+\frac{G^{* *}}{G^{*}}\right) y^{*}+\frac{G^{*}}{24 E^{2}} y \\
= & \frac{G^{*}}{4 E^{2}} \Omega_{\mathrm{DE}} \exp [-3(1+w) N],
\end{aligned}
$$

where a star denotes the derivative with respect to $N=$ $\log (a), \Omega_{\mathrm{DE}} \equiv \kappa \rho_{\mathrm{DE}} / 3 H_{0}^{2}$ is the dark energy fractional energy density today, and $w=$ const. is the usual darkenergy equation of state (EOS) parameter. Note that by writing in this way we have chosen to parameterize the background expansion to be the same as the dynamical dark-energy model $(w \neq-1)$ or the $\Lambda \mathrm{CDM}$ paradigm $(w=-1)$.

In the following, we shall assume $w=-1$ for simplicity and the calculations for general $w$ could be done similarly. In this case we have

$$
E=\Omega_{\mathrm{DE}}+\Omega_{m} \exp (-3 N)+\Omega_{r} \exp (-4 N),
$$

where $\Omega_{m}, \Omega_{r a}$ are respectively the fractional densities for non-relativistic and relativistic matter species. Deep into the radiation-dominated era we have $E \doteq \Omega_{r} \exp (-4 N)$, and thus Eq. (40) reduces to

$$
y^{* *}+9 y^{*}+8 y=48 \Omega_{\mathrm{DE}},
$$

whose solution is

$$
y(N)=A \exp (-N)+B \exp (-8 N)+6 \Omega_{\mathrm{DE}} .
$$

In this work we shall require

$$
\lim _{|G, R| \rightarrow \infty}\left|\frac{f(G)}{R}\right| \rightarrow 0
$$

so that $B$ could be set to zero in Eq. (43). To obtain the background evolution numerically, we start deep in the radiation-dominated era (e.g., at $\left.a=10^{-6}\right)$ and take the radiation-dominated solution, Eq. (43), to be the initial condition. The solutions to Eq. (40) are then characterized by a single parameter $A$. Different values of $A$ give the same background history, but in general lead to different evolutions for the perturbations, as we shall see below. Note that $A=0$ describes the standard $\Lambda \mathrm{CDM}$ paradigm.

Up to this point the procedure is quite similar to that in metric $f(R)$ gravity models. However, as was claimed above, the $f(G)$ gravity model cannot be used to reproduce arbitrarily parameterized background expansion histories. To explain why, we shall again take the $\Lambda \mathrm{CDM}$ background as an example and adopt the value $\Omega_{\mathrm{DE}}=0.76$, as suggested by the Wilkinson Microwave Anisotropy Probe (WMAP) three-year data 35]. Then it will be easy to find that $G^{*}$ changes its sign (from + to - ) at $N_{0} \approx-0.153$, which means that $G$ increases (decreases) when $N<(>) N_{0}$. As a result, with the match to $\Lambda \mathrm{CDM}$ at $N<N_{0}$ the function $f(G)$ with all possible values of $G$ has been determined, and there will generally be no freedom left to fix the evolution to $\Lambda \mathrm{CDM}$ at $N>N_{0}$ as well (of course, if $f(G)$ is a real cosmological constant then $\Lambda \mathrm{CDM}$ will also be reproduced at $N>N_{0}$, but in general reproducing $\Lambda \mathrm{CDM}$ on both sides of $N_{0}$ is far too strong a requirement to be satisfied). So, what we may conclude is that the $f(G)$ model can mimic a $\Lambda$ CDM universe up to $N_{0}$, after which the evolution might be governed by the already-determined $f(G)$. However, note that $N_{0} \approx-0.153$ corresponds to a 


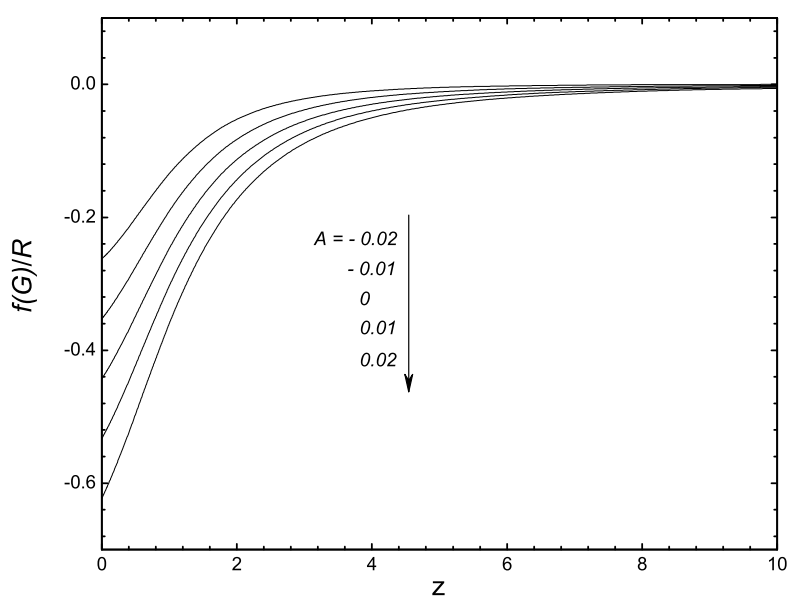

FIG. 1: The evolution versus redshift of $f(G) / R$ for the $f(G)$ gravity models fixed to match a $\Lambda \mathrm{CDM}$ cosmic expansion history $\left(\Omega_{\mathrm{DE}}=0.66\right)$. The curves from top to bottom are characterized by $A=-0.02,-0.01,0(\Lambda \mathrm{CDM}), 0.01,0.02$ respectively. Notice that with our convention $R<0$ and $f>0$.

critical redshift $z_{0} \approx 0.166$, so the transition from $\Lambda \mathrm{CDM}$ phase to a non- $\Lambda \mathrm{CDM}$ phase occurs quite late [36].

In the above, we have analyzed for a $\Lambda \mathrm{CDM}(w=-1)$ background. Nonetheless we can expect that similar situations exist for general dynamical $(w \neq-1)$ dark-energy backgrounds which are characterized by a late-time transition from $G^{*}>0$ to $G^{*}<0$. The investigation of background evolutions in general modified Gauss-Bonnet models is an interesting topic which is unfortunately beyond the scope of the present work. Here, we solve the perturbed equations numerically and investigate the effects of the $f(G)$ modifications to GR on the growth of linear perturbations. For simplicity we shall adopt a slightly unrealistic $\Lambda \mathrm{CDM}$ cosmic history which is described by $\Omega_{\mathrm{DE}}=0.66$ (in this case the transition from $G^{*}>0$ to $G^{*}<0$ has not taken place yet).

In Fig. 1, we show the redshift evolutions of $f(G)$ compared with $R$. Each curve here is characterized by a specific value of the coefficient $A$, which can be either positive, negative or 0 . Note that although all these curves lead to the same $\Lambda \mathrm{CDM}$ background evolution, not all of them are cosmologically viable. As will be discussed below, the stability of early-time perturbation growth requires $F_{G}<0$ (where $F_{G} \equiv \partial F / \partial G$ ) and that of small-scale perturbation growth furthermore requires $\left|F_{G}\right| H^{6} \ll 1$. The condition $F_{G}<0$ is found to correspond to the $A>0$ subclass of the solutions to Eq. (40). The evolution of $\left|F_{G}\right| H^{6}=-F_{G} H^{6}$ (which is dimensionless) in this subclass are shown in Fig. 2, It is obvious that $\left|F_{G}\right| H^{6}$ is a rapidly increasing quantity with respect to time whose magnitude is much smaller than 1 at early times.

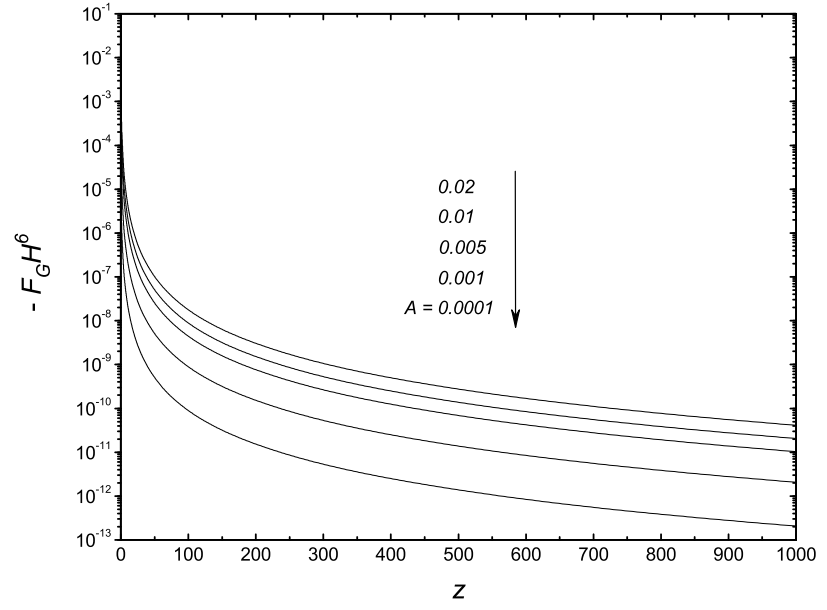

FIG. 2: The evolution versus redshift of the dimensionless quantity $-F_{G} H^{6}$ for the $f(G)$ gravity models fixed to match a $\Lambda$ CDM cosmic expansion history $\left(\Omega_{\mathrm{DE}}=0.66\right)$ which are stable in early time perturbation growths $\left(F_{G}<0\right)$. The curves from bottom to top are characterized by $A=$ $0.0001,0.001,0.005,0.01,0.02$ respectively. Note that a true cosmological constant has $F_{G}=0$.

\section{THE PERTURBATION EVOLUTION IN $f(G)$ MODELS}

Now we turn to the evolution of perturbation equations in the $f(G)$ model. The equations presented in Sec. II are not yet closed and from Eqs. (33- 36) we see that an evolution equation for $\hat{\nabla}_{a} F$ is also needed. To obtain this, let us look at the trace equation, Eq. (3) (which can also be obtained from Eq. (19) and the spatial derivative of Eq. (13)). Taking its spatial covariant derivative, after some manipulations we obtain the following evolution equation

$$
\begin{array}{r}
0=\ddot{\epsilon}+\left(\theta+\frac{2 \dot{\theta}}{\theta}\right) \dot{\epsilon}-S \\
+\left[\left(1+\frac{2 \dot{\theta}}{\theta^{2}}\right) \frac{k^{2}}{a^{2}}-\frac{4}{3}\left(\dot{\theta}+\frac{1}{3} \theta^{2}\right)-\frac{27}{16} \frac{1-\frac{4}{3} \dot{F} \theta}{\theta^{4} F_{G}}\right] \epsilon
\end{array}
$$

where $\epsilon$ is the harmonic expansion coefficient of $\hat{\nabla}_{a} F$, as

$$
\hat{\nabla}_{a} F=\sum_{k} \frac{k}{a} \epsilon Q_{a}^{k}
$$

and $Q_{a}^{k}=\frac{a}{k} \hat{\nabla}_{a} Q^{k}$ and $Q^{k}$ are the zero-order eigenvalues of the comoving Laplacian $a^{2} \hat{\nabla}^{2}\left(a^{2} \hat{\nabla}^{2} Q^{k}=k^{2} Q^{k}\right)$, $F_{G}=\partial F / \partial G=\partial^{2} f / \partial G^{2}$, and the source function $S$ is 
given by

$$
\begin{aligned}
S= & -\frac{3 \kappa}{4 \theta^{2}}\left(\mathcal{X}^{f}-3 \mathcal{X}^{p f}\right)-3\left(1-\frac{4}{3} \dot{F} \theta\right) \frac{\dot{\theta}}{\theta^{3}} \frac{k}{a} \mathcal{Z} \\
& -\frac{9}{2}\left(1-\frac{4}{3} \dot{F} \theta\right) \frac{\dot{\theta}}{\theta^{4}} \frac{k^{2}}{a^{2}} \eta-3\left(\frac{\ddot{F}}{\theta^{2}}-\frac{\dot{F}}{3 \theta}\right) \frac{k^{2}}{a^{2}} \eta \\
& -\left(\frac{2 \dot{F} \dot{\theta}}{\theta^{2}}+\frac{2 \ddot{F}}{\theta}+\frac{\dot{F}}{3}\right) \frac{k}{a} \mathcal{Z},
\end{aligned}
$$

where $\mathcal{X}^{f}, \mathcal{X}^{p f}, \mathcal{Z}, \eta$ are respectively the harmonic expansion coefficients of $\hat{\nabla}_{a} \rho^{f}, \hat{\nabla}_{a} p^{f}, \hat{\nabla}_{a} \theta$ and $\hat{\nabla}_{a} \hat{R}$ (see for example [31]). Moreover, here we are working in the CDM frame (with the 'observer' comoving with darkmatter particles and so free-falling) in which case we can set $A_{a}=0$ [30, 31] to simplify computations. In this case we have (up to first order)

$$
\begin{aligned}
\hat{\nabla}_{a} \dot{F} & =\sum_{k} \frac{k}{a} \dot{\epsilon} Q_{a}^{k}, \\
\hat{\nabla}_{a} \ddot{F} & =\sum_{k} \frac{k}{a} \ddot{\epsilon} Q_{a}^{k} .
\end{aligned}
$$

The presence of the term $-\frac{27}{16} \frac{1-\frac{4}{3} \dot{F} \theta}{\theta^{4} F_{G}}$ in Eq. (44) is no- table. As we have seen in the above section, the magnitude of the dimensionless quantity $\left|\theta^{6} F_{G}\right|$ is tiny at early times (deep into the matter- and radiation-dominated eras) so that at that time this term dominates over the other two in the squared brackets. If $F_{G}>0$, then $-\frac{27}{16} \frac{1-\frac{4}{3} \dot{F} \theta}{\theta^{4} F_{G}} \rightarrow-\infty$ at these early times, which makes the perturbation $\epsilon$ unstable and it grows quickly to spoil the linear theory. This is similar to the analysis of stability in $f(R)$ models [15, 16]. For the subclass of models with $F_{G}<0$, to which we are restricting ourselves, $-\frac{27}{16} \frac{1-\frac{4}{3} \dot{F} \theta}{\theta^{4} F_{G}} \rightarrow \infty$ and the value of $\epsilon$ quickly settles towards $-\frac{16}{27} \theta^{4} F_{G} S$. It can be checked easily that this is equivalent to $\hat{\nabla}_{a} F \doteq F_{G} \hat{\nabla}_{a} G$, which is as expected (note that at these times the influence of $f(G)$ corrections is negligible and all perturbation quantities except $\epsilon$ follow their standard GR evolution). Note that this could be used as the initial condition for $\epsilon$ when we evolve it numerically, and the initial condition for $\dot{\epsilon}$ could be obtained simply by taking its time derivative.

This is not the whole story. We could use Eq. (19) to substitute the term $-3 \frac{\dot{\theta}}{\theta^{3}} \frac{k}{a} \mathcal{Z}-\frac{9}{2} \frac{\dot{\theta}}{\theta^{4}} \frac{k^{2}}{a^{2}} \eta$ in Eq. (46) to re-express the evolution equation of $\epsilon$ as

$$
\ddot{\epsilon}+\left(\theta+\frac{4 \dot{\theta}}{\theta}\right) \dot{\epsilon}+\left[\left(1+\frac{4 \dot{\theta}}{\theta^{2}}\right) \frac{k^{2}}{a^{2}}-2\left(\dot{\theta}+\frac{\dot{\theta}^{2}}{\theta^{2}}+\frac{2}{9} \theta^{2}\right)-\frac{27}{16} \frac{1-\frac{4}{3} \dot{F} \theta}{\theta^{4} F_{G}}\right] \epsilon=S^{\prime}
$$

where

$$
\begin{aligned}
S^{\prime}= & -\frac{3 \kappa}{4 \theta^{2}}\left(\mathcal{X}^{f}-3 \mathcal{X}^{p f}\right)-3\left(\frac{\ddot{F}}{\theta^{2}}-\frac{\dot{F}}{3 \theta}\right) \frac{k^{2}}{a^{2}} \eta \\
& -\frac{9}{2} \frac{\dot{\theta}}{\theta^{4}} \kappa \mathcal{X}^{f}-\left(\frac{4 \dot{F} \dot{\theta}}{\theta^{2}}+\frac{2 \ddot{F}}{\theta}+\frac{\dot{F}}{3}\right) \frac{k}{a} \mathcal{Z} .
\end{aligned}
$$

The term $\left(1+\frac{4 \dot{\theta}}{\theta^{2}}\right) \frac{k^{2}}{a^{2}} \epsilon$ in Eq. (47) makes the situation more complicated. During the whole matter-dominated era and part of the deceleration-to-acceleration transition period we have $\left(1+\frac{4 \dot{\theta}}{\theta^{2}}\right)<0$. Thus, for the evolution of $\epsilon$ to be stable on small scales $\left(k^{2} / a^{2} H^{2} \gg 1\right)$, we must also require that this term is subdominant compared with the third term in the squared brackets; that is, $\left|F_{G} H^{6}\right|$ must be close enough to zero not only at early times, but also at low redshifts $(e . g ., z \lesssim \mathcal{O}(10))$. For example, in the $\Lambda \mathrm{CDM}$ limit $\left|F_{G} H^{6}\right|=0$, so that the small-scale instabilities will never appear. In general, since the deviation from $\Lambda \mathrm{CDM}$ in the $f(G)$ model is roughly characterized by the deviation of $A$ from zero, $A \ll 1$ should be satisfied in order that the model evades cosmological constraints from linear spectra.

To illustrate the effects discussed above, we have shown the evolution of some linear perturbation variables of the present model in Figs. 3 and 4 . Plotted in Fig. 3 are the evolutions of the (large-scale) Weyl potential $\phi_{k}$. This is the coefficient of the harmonic expansion of $\mathcal{E}_{a b}$ as $\mathcal{E}_{a b}=-\sum_{k} k^{2} \phi_{k} Q_{a b}^{k} / a^{2}$ and is related to the Newtonian potential, $\Psi$, by $\Psi=\phi-\kappa \Pi a^{2} / 2 k^{2}$ for any specified $k$-mode, where $\Pi$ is the anisotropic stress. From Eqs. (11, 33- 36), it is obvious that $\phi_{k}$ depends on $\epsilon$, and so from the analysis above it is easy to understand why on smaller scales $\phi_{k}$ changes so dramatically. This situation is quite similar to that in the Palatini $f(R)$ gravity model [18] where $f(R)=R+\alpha(-R)^{\beta}$ with $\beta>0$. Since the time evolution of $\phi_{k}$ determines the CMB power through the integrated Sachs-Wolfe (ISW) effect as

$$
I_{l}^{\mathrm{ISW}}=2 \int^{\tau_{0}} \phi_{k}^{\prime} j_{l}\left[k\left(\tau_{0}-\tau\right)\right] d \tau,
$$

where $j_{l}(k \tau)$ are the spherical Bessel functions, and $\tau_{0}$ the conformal time at present, the extremely rapid variations in $\phi_{k}$ might greatly enhance the angular power spectrum of temperature anisotropies, as in the the $\beta>0$ case of 


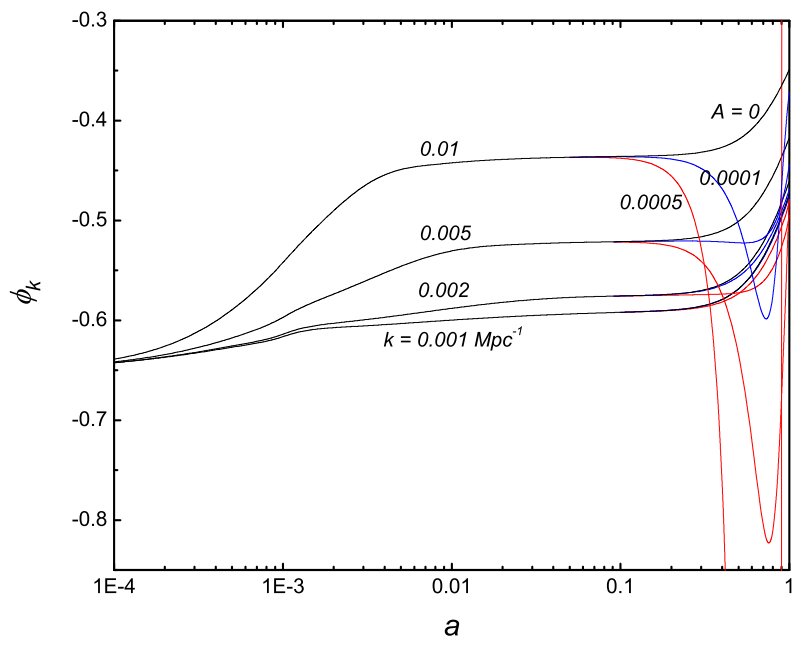

FIG. 3: (Color Online) Evolution of Weyl potential versus the cosmic scale factor $a$ at different scales, $k=$ $0.001,0.002,0.005,0.01 \mathrm{Mpc}^{-1}$ respectively from bottom to top . The values of $A$ are indicated beside the curves. $A=0$ corresponds to the $\Lambda$ CDM model.

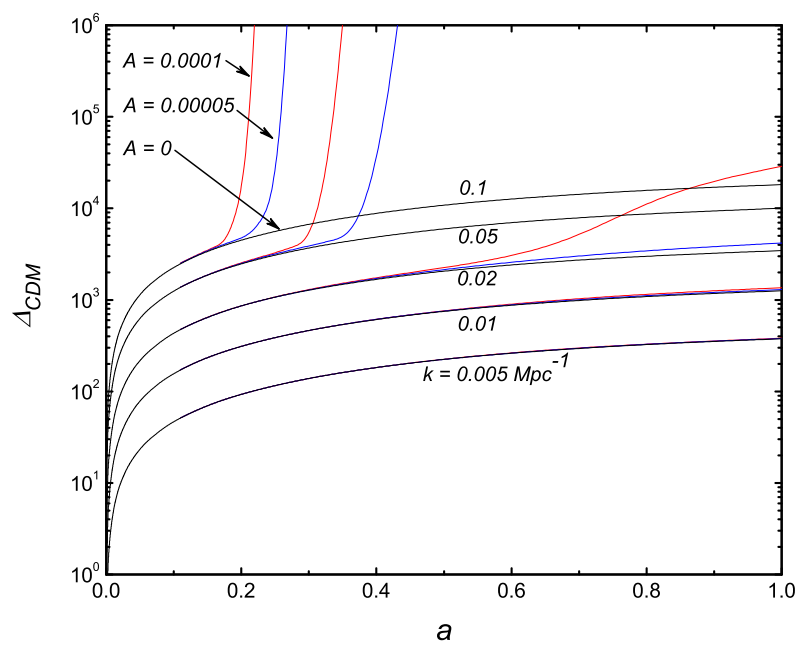

FIG. 4: (Color Online) Evolution versus cosmic scale factor $a$ of the cold dark matter density contrast $\Delta_{\mathrm{CDM}}$ at different scales, from bottom to top $k=$ $0.005,0.01,0.02,0.05,0.1 \mathrm{Mpc}^{-1}$ respectively. The values of $A$ are indicated beside the curves. $A=0$ corresponds to the $\Lambda$ CDM model. Note that the rapid growths of small-scale perturbations may make these scales leave the linear regime much earlier than in the standard $\Lambda$ CDM model.
Ref. [18]. This will provide the first stringent constraint on the present model (or equally on $A$ ).

In Fig. 4 we have displayed the time evolution of the cold dark matter density contrast, $\Delta_{\mathrm{CDM}}$, on different scales. As expected, on large scales the $k^{2}$ term in Eq. (47) never becomes important and $F_{G} H^{6}$ is small enough for our choices of $A$, so the deviation from $\Lambda \mathrm{CDM}$ $(A=0)$ is small. On small scales, however, the $k^{2}$ term is significant, and makes $\Delta_{\mathrm{CDM}}$ blow up quickly. This is also similar to the $\beta>0$ branch in Ref. [18], and produces a matter power spectrum which is strongly scale dependent for large $k$ (note the difference to metric $f(R)$ models [16]). This scale dependence will be strongly disfavored by current data on galaxy power spectra (such as those from Sloan Digital Sky Survey) and gives a second stringent constraint on the $f(G)$ cosmological models. Considering these, although evaluating the numerical constraint on $A$ is beyond the scope of the present work, we can claim that the parameter space for a viable $f(G)$ cosmology is highly limited. This, among others, once more reveals the difficulties appearing in explaining the cosmic acceleration with modified gravity models.

One should notice that the instabilities of the matter component found in these $f(G)$, and in $f(R)$ models within the Palatini approach, are in fact dependent on the nature of the dark matter and not only on the gravity sector 17]. For instance, instabilities are also found in GR models where cold dark matter is coupled to a light scalar field [37]. However, when dark matter is not cold, and so has a free-streaming length, instabilities might go away, as occurs in some interacting hot-dark-matterdark-energy models [38]. Hence, ruling out these gravity models just due to the instabilities in CDM might not be the last word.

\section{CONCLUSION}

To summarize: in this work we have consider the cosmology arising from a new modified gravity model, the modified Gauss-Bonnet model, where a function, $f(G)$, of the Gauss-Bonnet invariant is added to the EinsteinHilbert gravitational action to account for the current cosmic acceleration, at both the background and firstorder perturbation levels.

For the background-level evolution, we find that $f(G)$ models cannot describe arbitrarily parameterized cosmic histories leading to the current observations, because these histories are generally characterized by a transition from $\dot{G}>0$ to $\dot{G}<0$ which might impose too strong a requirement on the form of $f$.

For the linear-level evolution, we present the first-order perturbation equations for the $f(G)$ gravity model in the CGI formalism with a derivation which can also be applied to general $f\left(R, R^{a b} R_{a b}, R^{a b c d} R_{a b c d}\right)$ models. The special combination of $R, R^{a b} R_{a b}$ and $R^{a b c d} R_{a b c d}$ terms in the invariant $G$ ensures that the set of perturbation equations resemble that in the $f(R)$ gravity models and is 
much simpler than those in $f\left(R^{a b} R_{a b}\right)$ or $f\left(R^{a b c d} R_{a b c d}\right)$ theories. We analyze the perturbation equations and find that only the $\partial^{2} f / \partial G^{2}<0$ subclass of the models could have stable perturbation growth. Furthermore, even within this subclass, there will be a period during which the small-scale perturbation growth is no longer stable unless $\left|F_{G}\right| H^{6}$ is close enough to zero. This unstable growing period has two important consequences. Firstly, it makes the gravitational potential $\phi_{k}$ change very rapidly, which may greatly enhance the ISW effect and alter the CMB power. Secondly, the smallscale dark matter density perturbations grow much more quickly than in the $\Lambda \mathrm{CDM}$ paradigm, which might lead to a strongly scale-dependent matter power spectrum. In both cases, the model therefore faces stringent constraints from the cosmological data sets on linear spectra. We thus conclude that the parameter space for a viable $f(G)$ cosmological model is highly constrained.

There are several points which arise from this work but are beyond the scope of the present investigation. For example, it might be interesting to make an analysis of the evolution dynamics for general $f(G)$ models and to include the constraints arising from solar-system tests of gravity on the model.

\section{Acknowledgments}

We thank Prof. S. Odintsov for communications stimulating this work and Dr. Yong-Seon Song for helpful discussion. The numerical calculation of this work utilizes a modified version of the CAMB code [31, 39]. B. Li is supported by the Overseas Research Studentship, Cambridge Overseas Trust and the Department of Applied Mathematics and Theoretical Physics at the University of Cambridge. DFM acknowledges the Humboldt Foundation and the Research Council of Norway through project number $159637 / \mathrm{V} 30$.

Notes added: There has recently appeared another work [40] whose authors analyze a specific form of $f(R, G)$ theory by different methods. They also conclude that their model does not give a viable cosmology because of unstable behavior.
[1] E. J. Copeland, M. Sami and S. Tsujikawa, Int. J. Mod. Phys. D 15, 1753 (2006).

[2] J. D. Barrow and A. C. Ottewill, J. Phys. A: Math. Gen. 16, 2757 (1983).

[3] J. D. Barrow and S. Cotsakis, Phys. Lett. B 214, 515 (1988).

[4] K. I. Maeda, Phys. Rev. D 39, 3159 (1989).

[5] S. M. Carroll, A. De Felice, V. Duvvuri, D. A. Easson, M. Trodden, and M. S. Turner, Phys. Rev. D 71, 063513 (2005).

[6] D. A. Easson, Int. J. Mod. Phys. A 19, 5343 (2005).

[7] D. N. Vollick, Phys. Rev. D 68, 063510 (2003).

[8] G. Allemandi, A. Borowiec and M. Francaviglia, Phys. Rev. D 70, 043524 (2004).

[9] G. Allemandi, A. Borowiec and M. Francaviglia, Phys. Rev. D 70, 103503 (2004).

[10] G. Allemandi, A. Borowiec, M. Francaviglia and S. D. Odintsov, Phys. Rev. D 72, 063505 (2005).

[11] G. J. Olmo, Phys. Rev. Lett. 98, 061101 (2007). K. Kainulainen, V. Reijonen and D. Sunhede (2006), arXiv: gr-qc/0611132 E. Barausse, T. P. Sotiriou and J. C. Miller (2007), arXiv: gr-qc/0703132 K. Kainulainen, J. Piilonen, V. Reijonen and D. Sunhede (2007), arXiv: 0704.2729 [gr-qc].

[12] T. Chiba, Phys. Lett. B 575, 1 (2003); A. L. Erickcek, T. L. Smith and M. Kamionkowski (2006), [arXiv: astro-ph/0610483] I I. Navarro and K. V. Acoleyen (2006), [arXiv: gr-qc/0611127]; G. J. Olmo, Phys. Rev. Lett. 95, 261102 (2005); G. J. Olmo, Phys. Rev. D 72, 083505 (2005); G. J. Olmo, Phys. Rev. D 75, 023511 (2006); P. Zhang (2007), [arXiv: astro-ph/0701662]; W. Hu and I. Sawicki (2007), arXiv: 0705.1158 [astro-ph].

[13] S. Capozziello, V. F. Cardone and M. Francaviglia, Gen. Rel. Grav. 38, 711 (2006); M. Amarzguioui, O. El- garoy, D. F. Mota and T. Multamaki, Astron. Astrophys. 454, 707 (2006); T. P. Sotiriou, Class. Quant. Grav. 23, 1253 (2006); S. Fay, R. Tavakol and S. Tsujikawa (2007), [arXiv: astro-ph/0701479]; A. Borowiec, W. Godlowski and M. Szydlowski, Phys. Rev. D 74, 043502 (2006); K. Uddin, J. E. Lidsey and R. Tavakol (2007), arXiv: 0705.0232 [gr-qc].

[14] J. A. R. Cembranos, Phys. Rev. D 73, 064029 (2006); L. Amendola, D. Polarski and S. Tsujikawa (2006), [arXiv: astro-ph/0603703 arXiv: astro-ph/0605384; M. Fairbairn and S. Rydbeck (2007), [arXiv: astro-ph/0701900]; S. Capozziello, S. Nojiri, S. D. Odintsov and A. Troisi, Phys. Lett. B 639, 135 (2006); S. Nojiri and S. D. Odintsov, arXiv: hep-th/0601213 Phys. Rev. D 74, 086005 (2006); see also arXiv: hep-th/0611071, O. Mena, J. Santiago and J. Weller, Phys. Rev. Lett. 96, 041103 (2006); S. Fay, S. Nesseris and L. Perivolaropoulos, arXiv: gr-qc/0703006. R. Bean, D. Bernat, L. Pogosian, A. Silverstri and M. Trodden (2006), [arXiv: astro-ph/0611321]; L. Amendola, R. Gannouji, D. Polarski and S. Tsujikawa (2006), [arXiv: gr-qc/0612180]; T. Faulkner, M. Tegmark, E. Bunn and Y. Mao (2006), [arXiv: astro-ph/0612569]; L. Amendola and S. Tsujikawa (2007), arXiv: 0705.0396 [astro-ph]; S. Tsujikawa (2007), arXiv: 0705.1032 [astroph]; P. Zhang, M. Liguori, R. Bean and S. Dodelson (2007), arXiv: 0704.1932 [astro-ph]; O. Bertolami, C. G. Boehmer, T. Harko and F. S. N. Lobo, Phys. Rev. D 75, 104016 (2007); A. De Felice, A. Hindmarsh and M. Trodden, J. Cosmol. Astropart. Phys. 005, 0608 (2006); G. Calcagni, B. de Carlos and A. De Felice, Nucl. Phys. B 752, 404 (2006).

[15] Y. -S. Song, W. Hu and I. Sawicki, Phys. Rev. D 75, 044004 (2007). 
[16] B. Li and J. D. Barrow, Phys. Rev. D 75, 084010 (2007). [arXiv: gr-qc/0701111].

[17] T. Koivisto, Phys. Rev. D 73, 083517 (2006).

[18] B. Li, K. -C. Chan and M. -C. Chu, Phys. Rev. D 76, 024002 (2007). arXiv: astro-ph/0610794.

[19] B. Li and M. -C. Chu, Phys. Rev. D 74, 104010 (2006). [arXiv: astro-ph/0610486].

[20] S. Nojiri and S. D. Odintsov, Phys. Lett. B 631, 1 (2005).

[21] S. Nojiri, S. D. Odintsov and O. G. Gorbunova J. Phys. A: Math. Gen. 39, 6627 (2006).

[22] G. Cognola, E. Elizalde, S. Nojiri, S. D. Odintsov and S. Zerbini Phys. Rev. D 73, 084007 (2006).

[23] G. Cognola, E. Elizalde, S. Nojiri, S. D. Odintsov and S. Zerbini (2006), arXiv: hep-th/0611198.

[24] G. Cognola, M. Gastaldi and S. Zerbini (2007), arXiv: gr-qc/0701138

[25] S. Nojiri, S. D. Odintsov and M. Sasaki, Phys. Rev. D 71, 123509 (2005);

[26] B. M. N. Carter and I. P. Neupane, J. Cosmol. Astropart. Phys. 06, 004 (2006); Phys. Lett. B 638, 94 (2006).

[27] T. Koivisto and D. F. Mota, Phys. Lett. B 644, 104 (2007); Phys. Rev. D 75, 023518 (2007).

[28] G. F. R. Ellis and M. Bruni, Phys. Rev. D 40, 1804 (1989).

[29] G. F. R. Ellis and H. Van Helst (1998), in Theoretial and Observational Cosmology, edited by Marc Lachièze-Rey. pp. 1.

[30] A. Challinor and A. Lasenby, Astrophys. J. 513, 1 (1999).

[31] A. M. Lewis, PhD thesis, Queens' College and Astro- physics Group, Cavendish Lab., Cambridge University, 2000. http://www.mrao.cam.ac.uk/ aml1005/cmb.]

[32] J. -C. Hwang, Class. Quant. Grav. 7, 1613 (1990).

[33] B. Li, unpublished.

[34] By taking the spatial derivative of the relation $\nabla^{b} R_{a b}=$ $\frac{1}{2} \nabla_{a} R$, which results from the contracted Bianchi identity, and using Eqs. (27, 28), one can express $\Upsilon_{a}$ and $\Sigma_{a b}$ only in terms of $\hat{R}$ and kinematic quantities $\theta, A_{a}$ (plus their derivatives). In this way, the perturbation equations become higher $\left(4^{\text {th }}\right)$-order differential equations for these quantities only - as with traditional perturbation calculations. However, we think that the CGI perturbation equations, when are understood as propagation equations for the effective energy density, heat flux and istropic and unisotropic stresses, make the physics more transparent (and possibly easier to handle).

[35] D. N. Spergel et al. (2006), arXiv: astro-ph/0603449

[36] Needless to say, failing to reproduce the $\Lambda$ CDM cosmic history does not necessarily mean the failure of the model in general, as long as the background evolution can fit the supernovae data well.

[37] L. Amendola, Phys. Rev. D 62, 043511 (2000)

[38] A. W. Brookfield, C. van de Bruck, D. F. Mota and D. Tocchini-Valentini, Phys. Rev. Lett. 96, 061301 (2006).

[39] A. M. Lewis, A. Challinor and A. Lasenby, Astrophys. J. 538, 473 (2000). See also http://camb.info/.

[40] A. De Felice and M. Hindmarsh (2007), arXiv: 0705.3375 [astro-ph]. 